\title{
Diagnóstico de Fallas en Motores de Inducción mediante la Aplicación de Redes Neuronales Artificiales
}

\author{
Fernando Villada y Diego R. Cadavid \\ Facultad de Ingeniería, Departamento Ing. Eléctrica, Universidad de Antioquia, \\ Calle 67 No. 53-108, Oficina 20-105, Medellín-Colombia (fvillada@udea.edu.co)
}

\begin{abstract}
Resumen
En este trabajo se presenta un algoritmo para diagnosticar fallas entre espiras del estator de motores de inducción mediante la aplicación de redes neuronales artificiales (RNA). Los patrones de entrenamiento de las RNA son obtenidos a partir de un modelo de máquina que permite simular fallas internas bajo diferentes condiciones de carga y desequilibrio de tensión. Se muestra la implementación del método utilizando un analizador de redes eléctricas y un procesador digital de señales (DSP). Los resultados obtenidos experimentalmente en dos motores de 2 HP y 3 HP permiten concluir la fortaleza del algoritmo al permitir detectar fallas incipientes en motores de inducción y la factibilidad de implementación del mismo a nivel industrial.
\end{abstract}

Palabras clave: redes neuronales, motores de inducción, fallas en estator, diagnóstico

\section{Fault Diagnosis in Induction Motors using Artificial Neuronal Networks}

\begin{abstract}
A new algorithm to diagnose inter-turn faults in induction motors based on Artificial Neural Networks (ANN) is presented in this work. A machine model able to simulate internal faults under different load conditions and voltage unbalance was implemented and tested, in order to generate the training patterns of the ANN. An electrical network analyzer and a digital signal processor (DSP) are used to show the implementation of the method. Experimental results in a $2 \mathrm{HP}$ and $3 \mathrm{HP}$ induction motors show the robustness of the algorithm allowing detecting incipient faults and its implementation feasibility in industrial plants.
\end{abstract}

Keywords: artificial neural networks, induction motors, stator faults, diagnosis 


\section{INTRODUCCIÓN}

Los motores eléctricos de inducción son pieza fundamental en todo proceso industrial; su robustez, bajo costo, fácil mantenimiento y versatilidad los han hecho populares con aplicaciones que van desde artefactos caseros hasta equipos más sofisticados de tipo industrial. Sin embargo, estos motores tienen sus limitaciones y si son excedidos resultará en una falla prematura en el estator o rotor. Una de las fallas que ocurren en el estator es el cortocircuito entre espiras, la cual empieza con pocas espiras hasta llegar a una falla más severa. Para obtener información de la falla, la tendencia es monitorear la máquina con sensores de medida de tensión y corriente por ser de bajo costo y no invasivos.

Un importante indicador de falla es la corriente de secuencia negativa porque se mantiene aproximadamente constante para diferentes condiciones de carga, sin embargo, en la práctica este no es el caso porque la inherente asimetría de la máquina, la Instrumentación y el desbalance de tensión, pueden generar incrementos adicionales en dicha corriente. Los métodos propuestos en la literatura para sobrellevar esta dificultad están basados en tablas de búsqueda de datos y redes neuronales artificiales (RNA).

Di Stefano et al. (1997) desarrolló una red neuronal para diagnosticar fallas entre espiras. Los datos de entrenamiento fueron obtenidos mediante un modelo basado en la teoría de vectores espaciales. El indicador de falla utilizado fue el porcentaje de espiras falladas. Después de entrenar y validar la red, el error fue inferior a $2.4 \%$. Aunque el método es bueno, el inconveniente radica en las características del modelo por ser muy complejo en implementar.

Filippetti et al., (1996), presentó una arquitectura de red neuronal para detectar cortocircuitos en el devanado del estator. El indicador de falla es el porcentaje de espiras falladas. La estructura de la red fue el perceptrón multicapa con nueve neuronas en la capa oculta. Filippetti aunque propone la red, no presenta los resultados de la aplicación de la misma.

Tallam et al. (2001), propuso una RNA utilizando las componentes de secuencia de tensión y corriente para la detección de fallas entre espiras en un motor de inducción. La RNA estima la corriente de secuencia negativa, la cual es utilizada como indicador de falla. El inconveniente del método se tiene en la etapa de entrenamiento donde se requiere un volumen alto de datos experimentales, actividad que se dificulta en la práctica para cada uno de los motores en los que se implemente el sistema. En el siguiente trabajo de Tallam et al. (2003), se presenta un avance en el método anterior eliminando la etapa de aprendizaje y realizando el entrenamiento en línea. Sin embargo, cuando la falla evoluciona lentamente su detección se hace imposible.

En este trabajo se presenta un nuevo método para diagnosticar fallas en el estator de motores de inducción utilizando RNA. A pesar de utilizar la corriente de secuencia negativa como indicador de falla tal como lo presentaron Tallam et al. (2001, 2003); se elimina la dificultad práctica de obtener los datos experimentales dado que la mayor parte de los patrones de entrenamiento provienen de datos de simulación. Para tal efecto se implementa en Matlab y se verifica experimentalmente el modelo desarrollado por Arkan et al. (2005), considerando varias condiciones de carga y desequilibrio de tensión. Finalmente se muestra la implementación experimental mediante un prototipo diseñado para el monitoreo en línea del sistema y se presentan los resultados en dos motores de inducción de $2 \mathrm{Hp}$ y $3 \mathrm{Hp}$. Se demuestra la fortaleza del método al poder detectar fallas incipientes aún en los casos donde estas fallas evolucionen lentamente.

\section{CORRIENTE DE SECUENCIA NEGATIVA}

Las componentes de secuencia de tensión y corriente de un motor de inducción están dadas por (Kohler et al., 1992):

$$
\left[\begin{array}{c}
V p \\
V n \\
V o
\end{array}\right]=\left[\begin{array}{ccc}
Z p p & Z p n & Z p o \\
Z n p & Z n n & Z n o \\
Z o p & Z o n & Z o o
\end{array}\right] *\left[\begin{array}{c}
I p \\
I n \\
I o
\end{array}\right]
$$

Donde el índice $p, n$ y $o$, significan las secuencia positiva, negativa y cero de los fasores de tensión y corriente respectivamente. Zij representa la impedancia de secuencia $i$ debido a la secuencia $j$. 


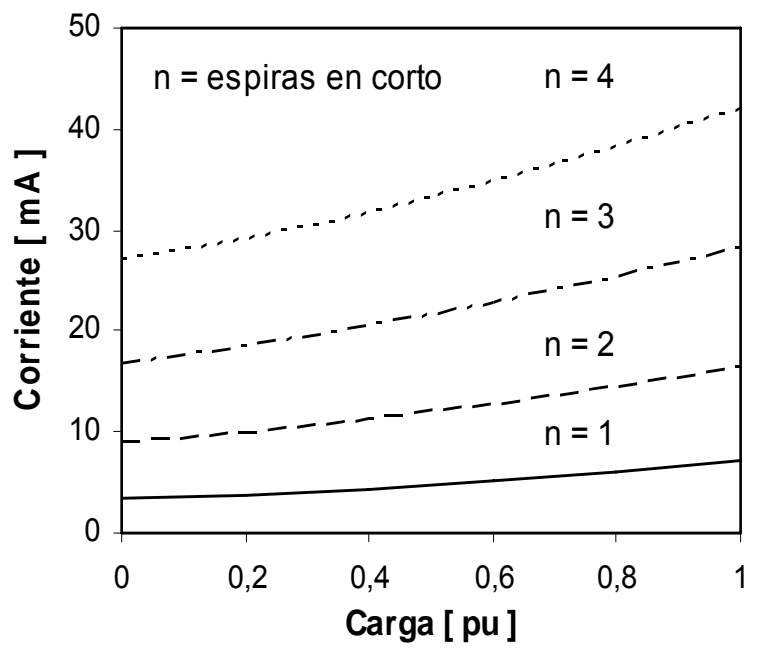

Fig.1: In versus el porcentaje de carga con impedancia de falla de $0.3 \mathrm{ohm}$.

Generalmente el motor es alimentado con tres fases sin neutro, por lo que la corriente de secuencia cero, lo, es cero, y la ecuación (1) se reduce a:

$\left[\begin{array}{c}I p \\ I n\end{array}\right]=\left[\begin{array}{ll}Z p p & Z p n \\ Z n p & Z n n\end{array}\right]^{-1} *\left[\begin{array}{c}V p \\ V n\end{array}\right]$

De la ecuación (2) se observa que In varía con los cambios en la fuente de potencia, las asimetrías del motor y la carga. Cuando la falla ocurre, las impedancias de la matriz cambian dado que el motor llega a ser asimétrico. Entonces, la falla, puede ser detectada comparando In (obtenida con el motor sano) con In_falla (obtenida con el motor fallado). La desviación en la corriente In, $\Delta / n$, es el indicador de falla y definida como:

$$
\Delta I n=I n \_ \text {falla }- \text { In }
$$

Realizando la simulación al motor A (ver anexo), la figura 1, muestra que $\ln$ es aproximadamente constante para diferentes condiciones de carga y se incrementa con el número de espiras en corto. La figura 2 muestra cómo varía la corriente de secuencia negativa en función del número de espiras en cortocircuito para diferentes valores de resistencia externa (limitadora de la falla). Se observa que hay una relación casi lineal entre In y el porcentaje de falla; dicha corriente es limitada por la impedancia de falla. La tabla 1 , ilustra cómo cambia In cuando se varía solamente la fuente de tensión de $0.5 \%$ a $3 \%$ de desbalance para un motor sin falla.

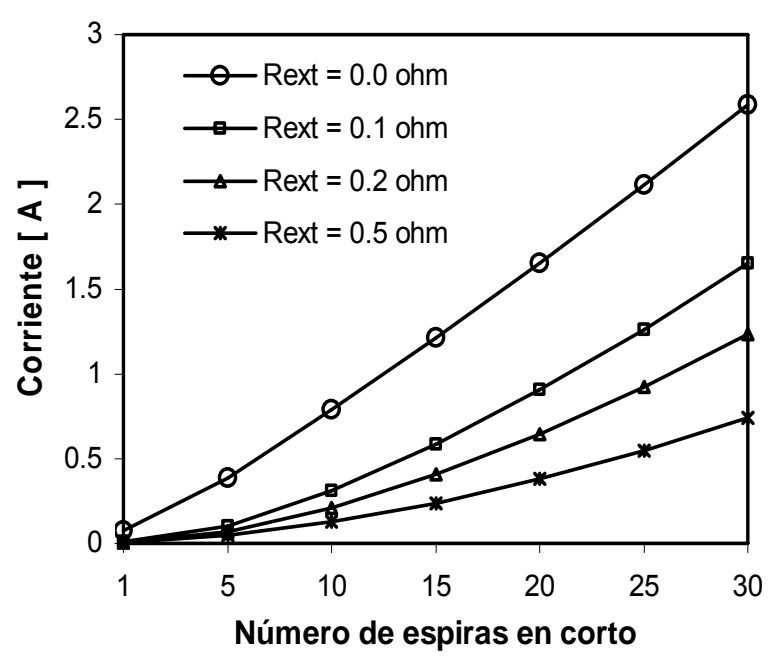

Fig. 2: In versus el número de espiras en corto, motor a plena carga.

Tabla 1: Variación de In para diferentes desbalances de tensión

\begin{tabular}{|c|c|c|}
\hline $\begin{array}{c}\text { Desbalance } \\
\text { tensión (\%) }\end{array}$ & $\begin{array}{c}\text { In } \\
(\mathrm{A})\end{array}$ & $\begin{array}{c}\text { Angulo de In } \\
\text { (grados) }\end{array}$ \\
\hline 0.5 & 0.078 & 124.67 \\
\hline 1.0 & 0.206 & -170.62 \\
\hline 1.5 & 0.230 & -77.46 \\
\hline 2.0 & 0.411 & -171.49 \\
\hline 2.5 & 0.370 & 142.62 \\
\hline 3.0 & 0.445 & 141.31 \\
\hline
\end{tabular}

Los anteriores resultados muestran que en general para un estado sin falla, In varía con los desbalances de tensión y las asimetrías en el motor.

\section{MODELO DE SIMULACIÓN DE FALLAS}

El modelo desarrollado por Arkan et al. (2005) fue implementado en este trabajo con el fin de obtener las componentes de secuencia de la corriente. El modelo se presenta en forma resumida en las ecuaciones 5 y 6 .

$$
\left[\begin{array}{c}
\lambda_{q}^{s h} \\
\lambda_{q}^{s} \\
\lambda_{d}^{s} \\
\lambda_{q}^{r} \\
\lambda_{d}^{r}
\end{array}\right]=\left[\begin{array}{lllll}
L_{q}^{s h} & L_{q}^{s s h} & 0 & L_{q}^{s h r} & 0 \\
L_{q}^{s h h} & L_{q}^{s} & 0 & L_{q}^{s r} & 0 \\
0 & 0 & L_{d}^{s} & 0 & L_{d}^{s r} \\
L_{q}^{s h r} & L_{q}^{s r} & 0 & L_{q}^{r} & 0 \\
0 & 0 & L_{d}^{s r} & 0 & L_{d}^{r}
\end{array}\right]\left[\begin{array}{l}
i_{q}^{s h} \\
i_{q}^{s} \\
i_{d}^{s} \\
i_{q}^{r} \\
i_{d}^{r}
\end{array}\right]
$$

Donde: sh hace referencia al devanado en cortocircuito, $r_{s h}$ es la resistencia del devanado en cortocircuito, $R_{\text {ext }}$ es la resistencia externa que limita la corriente de cortocircuito, 


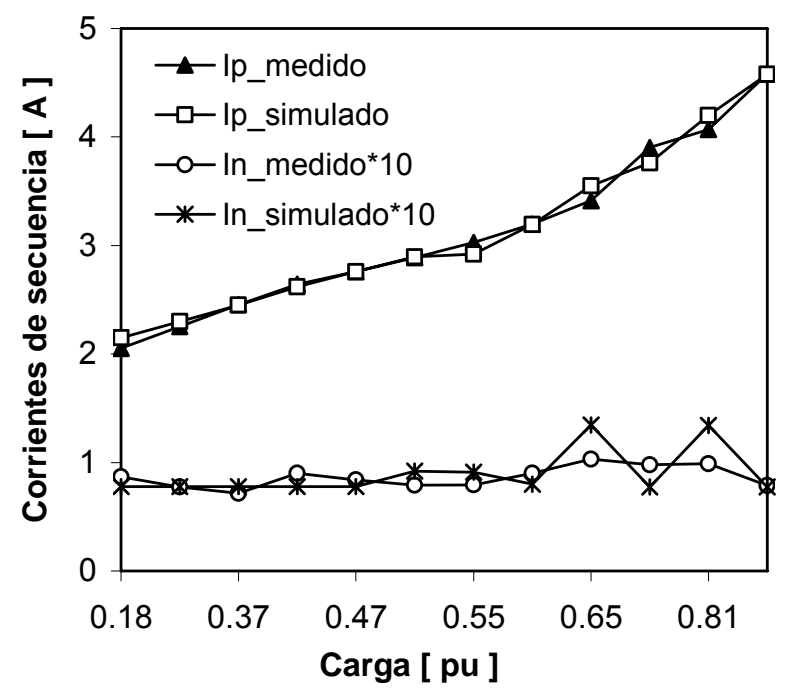

Fig. 3: Corrientes de secuencia positiva y negativa medidas y simuladas, motor de $2 \mathrm{Hp}$.

Con las siguientes ecuaciones de flujo:

$\lambda_{q}^{s h}=\int\left(v_{q}^{s h}-r_{s h} i_{q}^{s h}\right) d t$

$\lambda_{q}^{s}=\int\left(v_{q}^{s}-v_{q}^{s h}-r_{11}^{s} i_{q}^{s}-r_{12}^{s} i_{d}^{s}\right) d t$

$\lambda_{d}^{s}=\int\left(v_{d}^{s}-r_{21}^{s} i_{q}^{s}-r_{22}^{s} i_{d}^{s}\right) d t$

$\lambda_{q}^{r}=\int\left(w_{r} \lambda_{d}^{r}-r_{r}^{r} i_{q}^{r}\right) d t$

$\lambda_{d}^{r}=-\int\left(w_{r} \lambda_{q}^{r}+r_{r}^{r} i_{d}^{r}\right) d t$

$q$ y $d$ se refieren a ejes de cuadratura y directo respectivamente, $s$ y $r$ significan referido al estator y rotor respectivamente, $w_{r}$ es la velocidad del motor, y $\lambda$ es flujo.

El modelo se verificó experimentalmente, comparando las componentes de secuencia de la corriente para diferentes condiciones de carga y desbalance de tensión. Los resultados para un desbalance de tensión menor a $1 \%$ se ilustran en la figura 3 .

\section{MÉTODO PARA DIAGNÓSTICAR FALLAS}

El esquema de diagnóstico completo se ilustra en las figura 4 y figura 5 , el cual está dividido en dos etapas fundamentales: etapa de aprendizaje y etapa de monitoreo.

\section{Etapa de aprendizaje}

El objetivo de esta etapa es caracterizar los cambios de In en función de la carga y las variaciones de tensión. La red neuronal implementada es del tipo perceptrón multicapa (MPL) como se ilustra en la figura 6 . La red tiene 5 variables de entrada y 2 de salida. Esta red estima In para el motor sano bajo diferentes condiciones de carga y desequilibrio de tensión. El número de neuronas de la capa oculta se estima por ensayo y error hasta obtener la mejor configuración de la red.

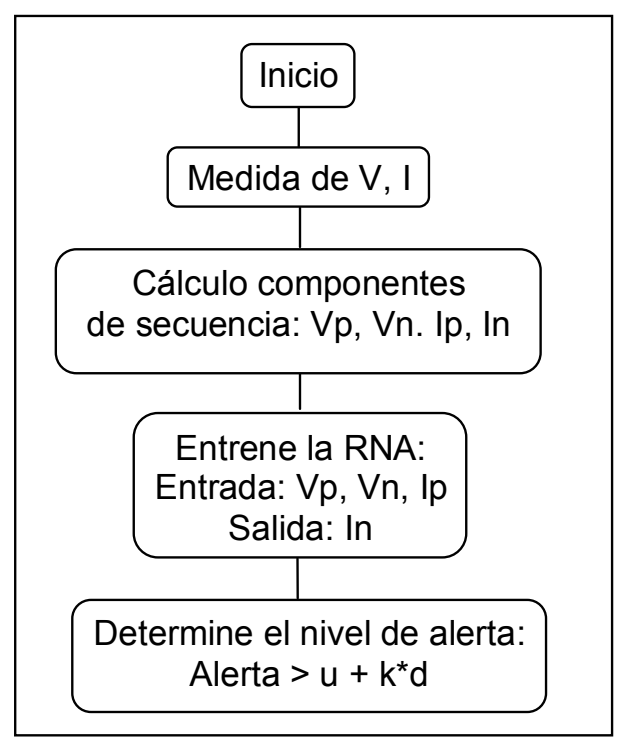

Fig. 4: Esquema de diagnóstico. Etapa de aprendizaje.

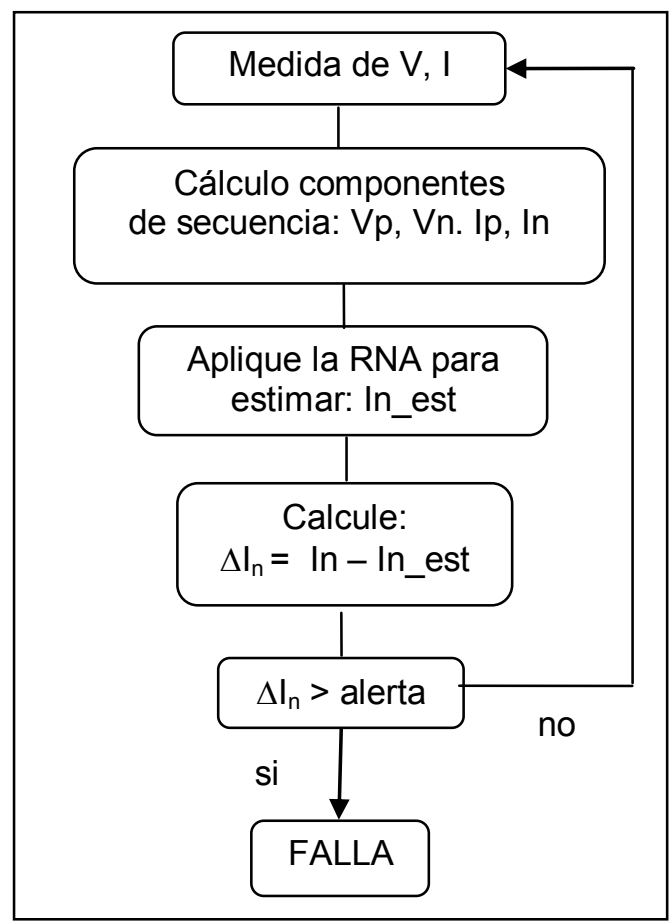

Fig. 5: Esquema de diagnóstico. Etapa de monitoreo. 


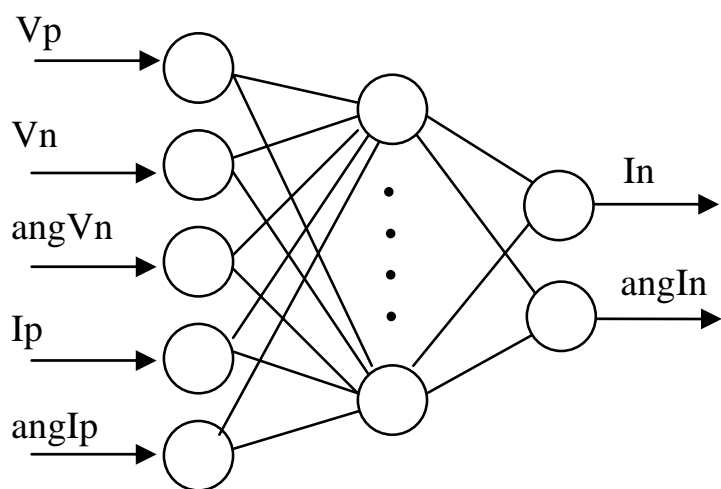

Fig. 6: Red neuronal implementada.

El esquema para determinar el indicador de falla $(\Delta / n)$ se ilustra en la figura 7 . El nivel de alerta se determina calculando la media, $u, y$ la desviación estándar, $d$, del indicador de falla $(\Delta I n)$; luego se calculan los niveles de alerta: $u+d, u+2 d$ y $u+3 d$. La sensibilidad para detectar la falla depende de estos límites; aproximadamente el $68 \%$ de los datos estarán bajo el límite $u+d$; el $95 \%$ de los datos estarán bajo el límite u+2d y el $99.7 \%$ de los datos estarán bajo el límite $u+3 d$.

\section{Etapa de monitoreo}

En la etapa de monitoreo se registran las tensiones y corrientes en los terminales del motor, se calculan las componentes de secuencia y se evalúa la red neuronal. Luego se calcula $\Delta I n$ y se compara con el nivel de alerta. Si se cumple que $\Delta I n$ es mayor que el nivel de alerta, se puede afirmar con algún nivel de confianza que hay falla (cortocircuito) en el sistema; en caso contrario, vuelve y se toman los registros de datos y se evalúa constantemente este indicador.

Cuando el monitoreo se hace en línea, la evolución o el control del indicador de falla se puede hacer a través de gráficas de control CUSUM en línea (Leger et al., 1998). En este caso, el objetivo es detectar cuando el indicador de falla tiene un corrimiento de su valor normal o límite.

\section{APLICACIÓN DEL MÉTODO}

\section{Aplicación mediante simulación}

En la simulación, se tomaron 1000 datos aleatorios de la tensión en cada fase, de los cuales: 766 se utilizaron para el entrenamiento, 109 para la evaluación y 125 para la prueba. La carga del motor se varió desde vacío a plena carga. El modelo propuesto por Arkan et al., (2005) se implementó para obtener todas las variables eléctricas del motor sin falla y con falla.

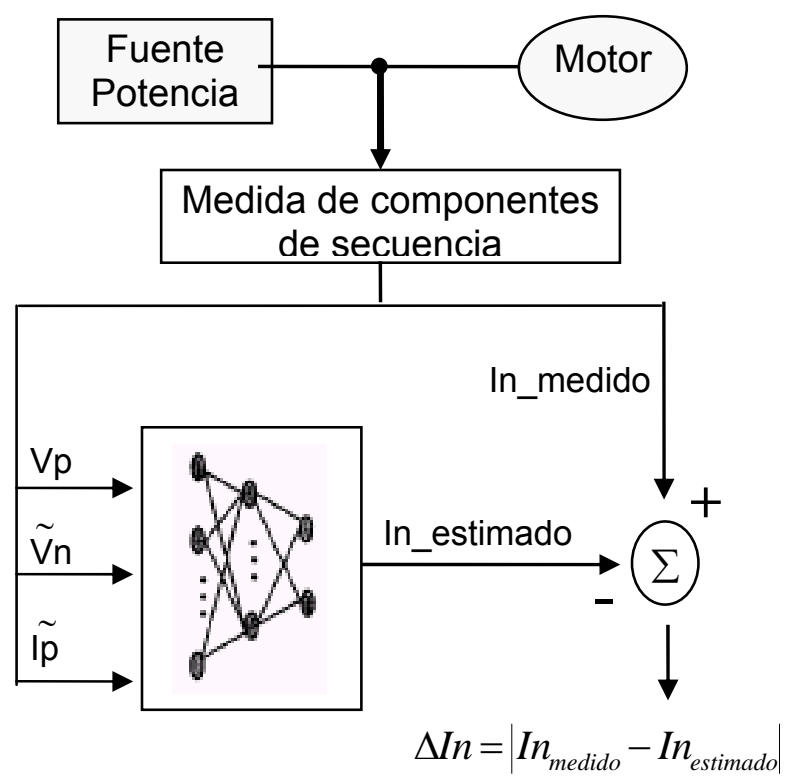

Fig. 7: Determinación del indicador de falla.

Los algoritmos de entrenamiento aplicados fueron las variantes del método de retropopagación (backpropagation) del toolbox de Matlab (Demuth y Beale, 1998): Levenberg -Marquardt (trainlm) y LevenbergMarquardt Optimization (trainbr).

En la tabla 2, se observa el error de entrenamiento de la red neuronal, la media y la desviación estándar del indicador de falla $\Delta / n$. La topología de la red seleccionada fue la 5-2 porque presenta el menor error en la media y desviación estándar. Los límites de alarma se observan en la figura 8 . La aplicación de la RNA con falla se ilustra en la figura 9 , con diferentes resistencias externas limitadoras de falla.

Aplicación experimental utilizando analizador de redes

El montaje experimental utilizado para la validación del modelo y la verificación del esquema de diagnóstico se muestra en la figura 10. Durante el registro de las variables eléctricas del motor a plena carga, se varió la fuente de potencia con pequeños desequilibrios de tensión (menor a 3\%) simulando las variaciones típicas reales. Se registraron 861 datos. 
Tabla 2. Resultados de la simulación del entrenamiento de la red neuronal para diferentes condiciones de carga

\begin{tabular}{|c|c|c|c|c|c|c|c|c|}
\hline \multicolumn{2}{|c|}{} & \multicolumn{4}{|c|}{ Algoritmos de entrenamiento derivados del método retropropagación } \\
\hline \multirow{2}{*}{$\begin{array}{c}\text { Capa } \\
\text { entrada }\end{array}$} & \multirow{2}{*}{$\begin{array}{c}\text { Capa } \\
\text { oculta }\end{array}$} & $\begin{array}{c}\text { Capa } \\
\text { salida }\end{array}$ & $\begin{array}{c}\text { Trainbr } \\
\text { entrenamiento }\end{array}$ & $\begin{array}{c}\text { Media } \\
\text { de } \Delta \mathrm{ln}\end{array}$ & $\begin{array}{c}\text { Desviación } \\
\text { estándar de } \\
\Delta \mathrm{ln}\end{array}$ & $\begin{array}{c}\text { Error de } \\
\text { entrenamiento }\end{array}$ & $\begin{array}{c}\text { Media } \\
\text { de } \Delta \mathrm{ln}\end{array}$ & $\begin{array}{c}\text { Desviación } \\
\text { estándar de } \\
\Delta \mathrm{ln}\end{array}$ \\
\hline 5 & 0 & 2 & 0.009972 & 0.0004017 & 0.0002315 & 0.08311 & 0.1076 & 0.0656 \\
\hline 5 & 3 & 2 & 0.001000 & 0.0005477 & 0.0003639 & 18.4402 & 0.4356 & 0.2082 \\
\hline 5 & 5 & 2 & 0.002000 & 0.0009468 & 0.0006384 & 5.0135 & 0.3681 & 0.2485 \\
\hline 5 & 10 & 2 & 0.000997 & 0.0006154 & 0.0005063 & 3.5448 & 0.3703 & 0.2315 \\
\hline
\end{tabular}

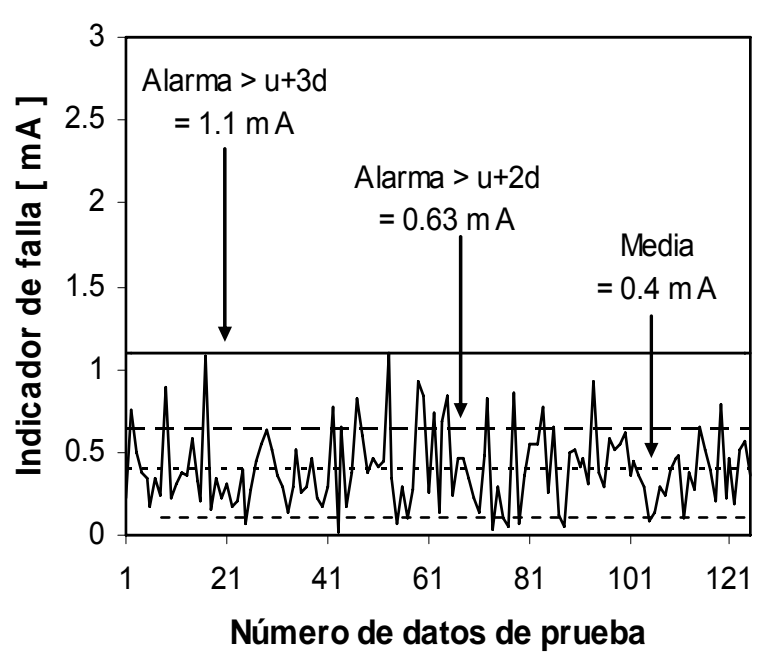

Fig. 8: Límites de alarma para motor de $2 \mathrm{Hp}$.

Luego de terminar los registros, se procedió a realizar los ensayos de cortocircuito. Para ello se utilizaron resistencias externas para limitar la corriente de cortocircuito.

Después de entrenar y evaluar la red neuronal, se seleccionó la red con estructura: 5-10-2. La tabla 3, ilustra los resultados. El límite de alarma y la aplicación de la red neuronal para varias espiras en corto se ilustran en la figura 11 y figura 12 respectivamente.

\section{Aplicación experimental utilizando prototipo digital}

Para medir las componentes de secuencia en línea, diseñamos un prototipo utilizando un procesador de señales digitales (DSP por sus siglas en ingles), cuyas componentes principales se ilustran en la figura 13.

El sensado de la corriente se hizo con pinzas amperimétricas marca fluke de $100 \mathrm{mV} / \mathrm{A}$.
El sensado de la tensión se hizo con un divisor de tensión resistivo y amplificadores de aislamiento de AD202, estos dispositivos proporcionan aislamiento galvánico de $2000 \mathrm{~V}$ y una distorsión máxima de $0.25 \%$.

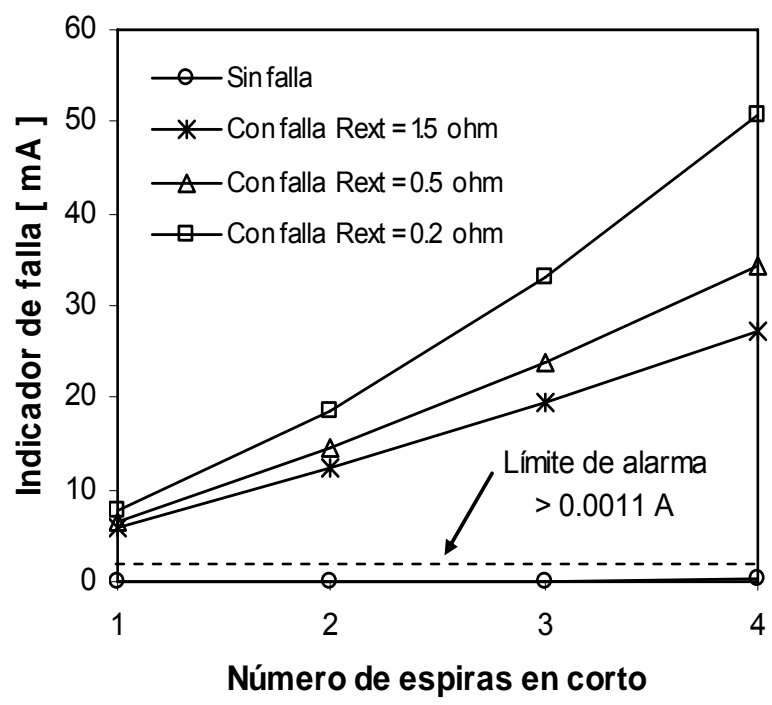

Fig. 9. Aplicación de la RNA con falla entre espiras. Motor de $2 \mathrm{Hp}$ a plena carga.

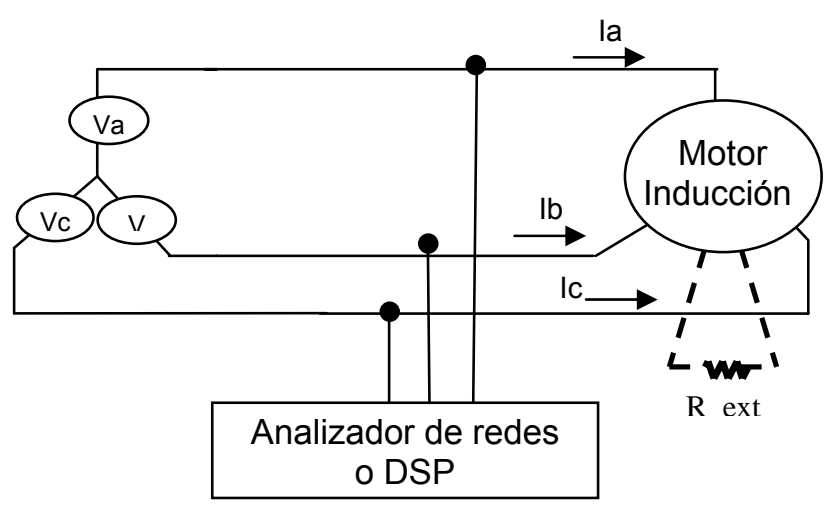

Fig. 10: Montaje experimental.

El sistema de acondicionamiento adecua las señales provenientes de los sensores de tensión y corriente para ser capturadas en forma adecuada por el conversor análogo

Información Tecnológica - Vol. 18 No 2 - 2007 
Tabla 3: Resultados experimentales del entrenamiento de la red neuronal, motor a plena carga

\begin{tabular}{|c|c|c|c|c|c|c|c|c|}
\hline \multicolumn{2}{|c|}{} & \multicolumn{3}{|c|}{ Algoritmos de entrenamiento derivados del método de retropropagación } \\
\hline \multirow{2}{*}{$\begin{array}{c}\text { Capa } \\
\text { entrada }\end{array}$} & $\begin{array}{c}\text { Capa } \\
\text { oculta }\end{array}$ & $\begin{array}{c}\text { Capa } \\
\text { salida }\end{array}$ & $\begin{array}{c}\text { Error de } \\
\text { entrenamiento }\end{array}$ & $\begin{array}{c}\text { Media } \\
\text { de } \Delta \mathrm{ln}\end{array}$ & $\begin{array}{c}\text { Desviación } \\
\text { estándar de } \\
\Delta I n\end{array}$ & $\begin{array}{c}\text { Error de } \\
\text { entrenamiento }\end{array}$ & $\begin{array}{c}\text { Media } \\
\text { de } \Delta \text { In }\end{array}$ & $\begin{array}{c}\text { Desviación } \\
\text { estándar de } \\
\Delta I n\end{array}$ \\
\hline 5 & 0 & 2 & 0.006176 & 0.00312 & 0.00169 & $3.64 \mathrm{E}-05$ & 0.0092 & 0.0050 \\
\hline 5 & 3 & 2 & 0.006162 & 0.00279 & 0.00176 & $1.42 \mathrm{E}-05$ & 0.0060 & 0.0036 \\
\hline 5 & 5 & 2 & 0.00492 & 0.00270 & 0.00175 & $1.31 \mathrm{E}-05$ & 0.0034 & 0.0058 \\
\hline 5 & 10 & 2 & 0.003728 & 0.002785 & 0.00199 & $1.21 \mathrm{E}-05$ & 0.0061 & 0.0064 \\
\hline
\end{tabular}

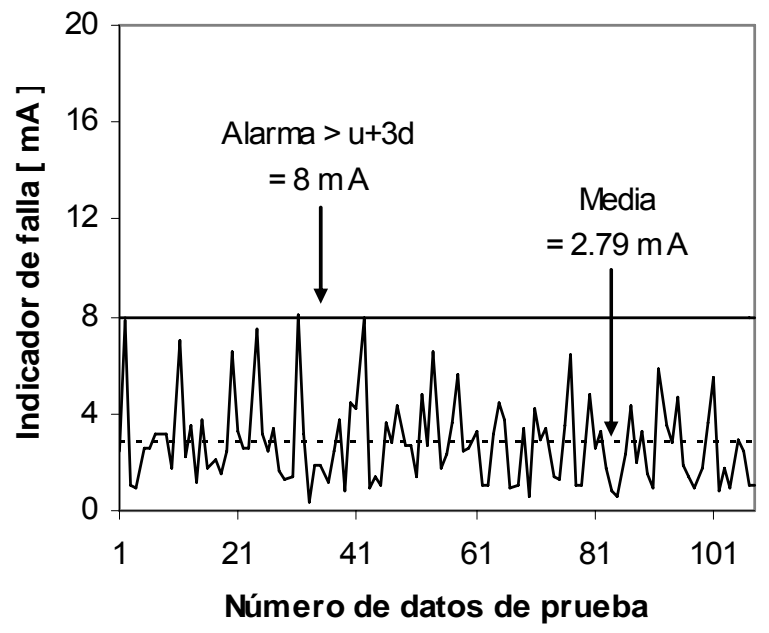

Fig. 11: Desempeño del indicador de falla $(\Delta l n)$ sin falla. Motor de $2 \mathrm{Hp}$ a plena carga.

digital (ADC) en la DSP. Una de las adecuaciones es el filtrado de altas frecuencias para cumplir con el teorema del muestreo de Nyquist. La DSP se encarga de configurar y ejecutar la captura de las señales de tensión y corriente, procesar los datos capturados y realizar la comunicación con el PC por medio de una interfaz serial RS-232. EI PC hace las veces de Interfaz de usuario con el prototipo. Por medio de una interfaz gráfica en Matlab el usuario puede configurar diversos parámetros de la captura de señales, visualizar y salvar los resultados generados en la DSP.

En esta aplicación se utilizó un motor de $3 \mathrm{Hp}$ (motor B), jaula de ardilla, conexión doble estrella, como se ilustra en el anexo. Después de entrenar la RNA con datos simulados y medidos se encontró que la mejor estructura correspondió a una red 5-2. Luego se realizaron varios cortocircuitos sin limitación de resistencias externas. La impedancia de falla fue aproximadamente de $0.088 \Omega$. Los resultados se ilustran en la figura 14.

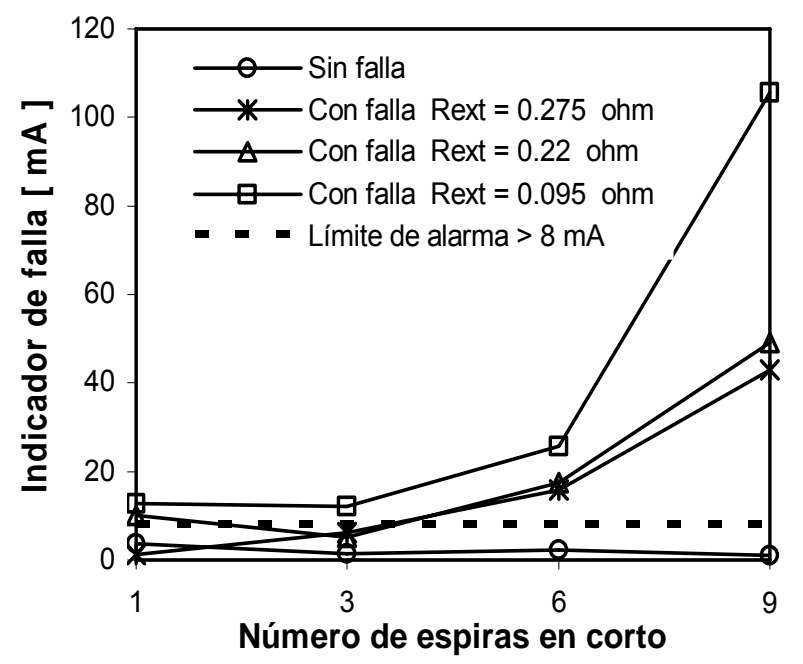

Fig. 12: Aplicación de la red neuronal 5-10-2 con falla entre espiras y datos experimentales. Motor de $2 \mathrm{Hp}$ a plena carga.

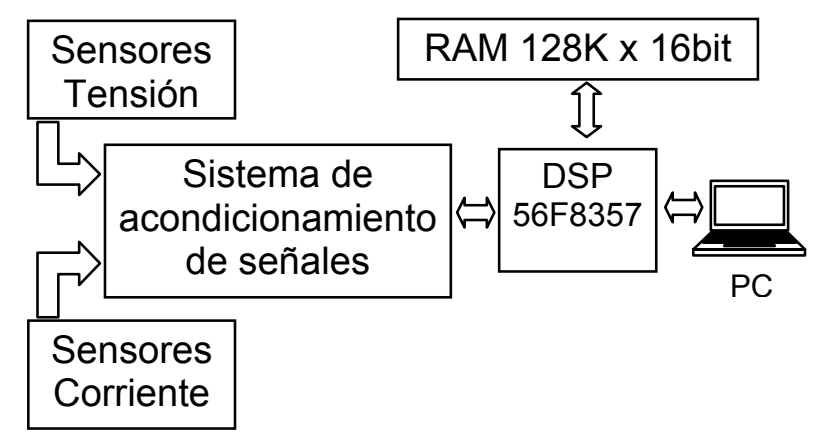

Fig. 13: Arquitectura del prototipo implementado

Los resultados anteriores muestran que la evaluación de la red neuronal con los datos de prueba establece el marco de referencia para determinar los límites de alarma de posibles fallas. Estos límites dependen de la precisión de la red neuronal, permitiendo detectar de forma confiable fallas a partir de dos espiras. 


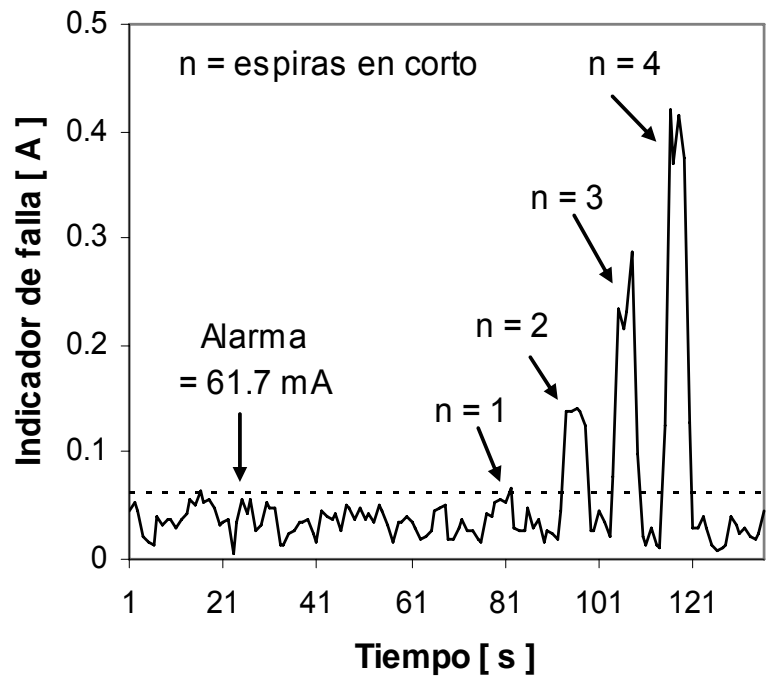

Fig. 14: Implementación experimental en prototipo utilizando DSP. Motor de $3 \mathrm{Hp}$ a $82 \%$ de carga y fallas entre espiras.

\section{ANEXO}

Parámetros de los motores utilizados en los experimentos

\begin{tabular}{|c|c|}
\hline $\begin{array}{c}\text { Motor A - Estrella } \\
\text { simple }\end{array}$ & $\begin{array}{c}\text { Motor B - estrella } \\
\text { Doble }\end{array}$ \\
\hline $\begin{array}{l}\text { Potencia }=2 \mathrm{Hp} \\
V=220 \mathrm{Vrms} \\
f=60 \mathrm{~Hz} \\
\mathrm{P}=4 \\
\mathrm{~J}=0.03 \mathrm{~kg} \cdot \mathrm{m}^{2} \\
\mathrm{~N}=256 \text { espiras por } \\
\text { fase } \\
\mathrm{Xs}=1.998 \Omega \\
\mathrm{Xr}=1.998 \Omega \\
\mathrm{Xm}=63.168 \Omega \\
\mathrm{Rs}=1.414 \Omega \\
\mathrm{Rr}=0.947 \Omega\end{array}$ & $\begin{array}{l}\text { Potencia }=3 \mathrm{Hp} \\
\mathrm{V}=220 \mathrm{Vrms} \\
\mathrm{f}=60 \mathrm{~Hz} \\
\mathrm{P}=4 \\
\mathrm{~J}=0.04 \mathrm{~kg} \cdot \mathrm{m}^{2} \\
\mathrm{~N}=102 \text { espiras por } \\
\text { cada camino en } \\
\text { paralelo } \\
\mathrm{Xs}=1.5068 \Omega \\
\mathrm{Xr}=1.5068 \Omega \\
\mathrm{Xm}=27.810 \Omega \\
\mathrm{Rs}=0.720 \Omega \\
\mathrm{Rr}=0.7817 \Omega\end{array}$ \\
\hline
\end{tabular}

\section{CONCLUSIONES}

En este trabajo se presentó un nuevo método para diagnosticar fallas en el estator de motores de inducción utilizando RNA. Para tal efecto, se implementó en Matlab y se verificó experimentalmente un modelo de motor de inducción que permite simular el comportamiento del motor bajo diferentes condiciones de carga y desequilibrio de tensión. Se mostró la facilidad de aplicación del sistema en la industria mediante un analizador de redes eléctricas o utilizando un prototipo digital. Los resultados experimentales demuestran la fortaleza del método al poder detectar de forma confiable fallas incipientes, aún en los casos donde dichas fallas evolucionen lentamente.

\section{REFERENCIAS}

Arkan, M., D. Kostic-Perovic y P. J. Unsworth, Modelling and simulation of induction motors with inter-turn faults for diagnostics, Electric Power Systems Research, 75, 57-66 (2005).

Demuth, H. y M. Beale, User's guide for neural network toolbox for use with MATLAB. Natick, MA: The MathWorks Inc., 1998.

Di Stefano, R., S. Meo y M. Scarano, Induction motor faults diagnostic via artificial neural network (ANN), International Symposium Industrial Electronics, ISIE'94, 220-225 (1994), Santiago -Chile.

Filippetti, F., G. Franceschini, A. Ometto y S. Meo, A survey of neural network approach for induction machine on-line diagnosis, in Proc. UPEC'96, Iraklio, Greece, 1, 17-20 (1996).

Leger, R. P., Wm. J. Garland y W. F. S. Poehlman, Fault detection and diagnosis using statistical control charts and artificial neural networks, Artificial Intelligence in Engineering, 12 (1998) 35-47.

Tallam, R.M., Habetler, T.G. y Harley, R.G., Continual On-line training of neural networks with applications to electric machine fault diagnostics, in Proc. IEEE $32^{\text {nd }}$ Power Electronics Specialists Conf., 4, 2224-2228 (2001).

Tallam, R.M., Lee, S.B., Stone, G., Kliman, G.B., Yoo, J., Habetler, T.G., Harley, R.G., A survey of methods for detection of stator related faults in induction machines, $4^{\text {th }}$ IEEE International Symposium on Diagnostic for Electric machines, Power Electronics and Drives, SDEMPED 2003, 35-46 (2003). 